\title{
Effect of high blood pressure on thyroid and lipid profile of pregnant women
}

\section{Gurpreet Kaur Gill*, Mandeep Kaur, Partapbir Singh, Juhi Kataria}

Department of Medical Lab Sciences, Khalsa College of Pharmacy and Technology, Amritsar, Punjab, India

Received: 28 June 2019

Accepted: 31 July 2019

*Correspondence:

Dr. Gurpreet Kaur Gill,

E-mail: gurpreet_pau25@yahoo.com

Copyright: (C) the author(s), publisher and licensee Medip Academy. This is an open-access article distributed under the terms of the Creative Commons Attribution Non-Commercial License, which permits unrestricted non-commercial use, distribution, and reproduction in any medium, provided the original work is properly cited.

\begin{abstract}
Background: Hypertension is common medical problem encountered during pregnancy, complicating up to $10 \%$ pregnancies. Hypertension is defined as the systolic blood pressure $\leq 140 \mathrm{mmHg}$ and a diastolic blood pressure $\geq 90$ $\mathrm{mmHg}$ on two separate measurements at least 4-6 hours apart. During pregnancy thyroid demand changes with increased iodine uptake and synthesis of thyroid hormone. Serum from pre-eclamptic women had both a higher ratio of free fatty acid to albumin and increase up take of free fatty acids, which are further esterified to triglyceride. The aim of the present study was to evaluate thyroid hormone, lipid profile and urine albumin in hypertensive pregnant women and its comparison with normotensive pregnant women

Methods: Total 90 samples were collected from Beri Maternity Hospital and Civil Hospital, Amritsar. Blood samples were analysed for biochemical parameters viz. lipid profile and thyroid profile. Urine samples were also analysed for presence of albumin.

Results: From the study it has been revealed that thyroid stimulating hormone (TSH) levels increased in hypertensive pregnant women mean $(4.26 \pm 1.1 \mu / \mathrm{ml})$. The total cholesterol $(234.56 \pm 14.2 \mathrm{mg} / \mathrm{dl})$, triglyceride $(138 \pm 16.96 \mathrm{mg} / \mathrm{dl})$, HDL $(50.7 \pm 7.4 \mathrm{mg} / \mathrm{dl})$, LDL $(156.2 \pm 17.11 \mathrm{mg} / \mathrm{dl})$ and VLDL $(27.7 \pm 3 \mathrm{mg} / \mathrm{dl})$ was found altered in hypertensive pregnancy as compared to normotensive pregnancy. The albumin excretion was seen in $13.3 \%$ hypertensive pregnancies.

Conclusions: TSH were elevated in hypertensive pregnant women due to the effects of estrogen. Among all the parameters of lipid profile, total cholesterol, TG, LDL and VLDL has been increased significantly in hypertensive pregnant women as compared to normotensive pregnant women due endothelial dysfunction.
\end{abstract}

Keywords: Hypertension, Lipid profile, Pregnancy, TSH, Urine albumin

\section{INTRODUCTION}

Hypertensive disorders in pregnancy may cause maternal and fetal morbidity and a common reason of maternal mortality. Hypertension in pregnancy is defined as a systolic blood pressure $\geq 140 \mathrm{~mm} \mathrm{Hg}$ and a diastolic blood pressure $\geq 90 \mathrm{~mm} \mathrm{Hg}$ on two separate measurements at least 4-6 hours apart after 20 weeks of gestation in women. ${ }^{1}$ Hypertension in pregnancy complicates $5-10 \%$ of all pregnancies worldwide whereas pregnancy-induced hypertension $(\mathrm{PIH})$ has been identified in $3.9 \%$ of all pregnancies. ${ }^{2}$ When hypertension is first identified during a woman's pregnancy and she is at less than 20 weeks' gestation, blood pressure elevations usually represent chronic hypertension. In contrast, new onset of elevated blood pressure readings after 20 weeks gestation is gestational hypertension which may further lead to preeclampsia if uncontrolled. Preeclampsia occurs 
in $3-6 \%$ of all pregnancies and the incidence is 1.5 to 2 times higher in first time pregnancies. ${ }^{3}$ Pre-eclampsia is a multisystem disease unique to human pregnancy characterised by hypertension and organ system derangement. Pre-eclampsia can severely complicate the pregnancy and it is in the top three causes of maternal morbidity and mortality globally. It is the leading cause of fetal growth restriction, intrauterine fetal demise and planned preterm birth. ${ }^{4}$ Pre-eclampsia occurs after 20 weeks gestation and is classically defined as a triad of hypertension, edema and proteinuria. ${ }^{5}$ Proteinuria is one of the cardinal features of preeclampsia. Pregnancy induces complex changes in circulating maternal steroid hormones and in thyroid binding globulin (TBG) concentrations. Plasma clearance of the protein gets reduced by changes in TBG glycolsylation.

Thyroid dysfunction without any primary thyroid disease may result in various qualitative changes of triglycerides, phospholipids, cholesterol, and other lipoproteins. ${ }^{6}$ Dyslipidemia, a consequence of thyroid dysfunction, generally increase the risk of cardiovascular disease. ${ }^{7}$ The influence of TSH on lipid profile has been assumed to be mediated indirectly, through its effect on thyroid hormones. ${ }^{8}$ An abnormal lipids profile is known to be strongly associated with atherosclerotic cardiovascular diseases and has a direct effect on endothelial dysfunction. Elevated insulin resistance occurring in preeclampsia would increase fatty acid mobilization from visceral fat. It promote over production of VLDL by liver and suppression of post hepatic lipoprotein lipase which could increased serum free fatty acids. The similarity between the lesions of preeclampsia and atherosclerosis had led to speculation of a common pathophysiological pathway. Women who developed preeclampsia showed even more dramatic lipid alterations. Most show a preeclampsia-dyslipidemic pattern of increased triglycerides, cholesterol, low density lipoprotein (LDL), decreased high density lipoprotein (HDL) concentration. ${ }^{9}$ It is imperative to study the association of lipid alterations with high blood pressure in pregnancy so that these could be managed to avoid any maternal cardiovascular risk. Thus the current investigation was planned to evaluate the level of TSH and lipid profile in hypertensive pregnancy and its comparison with normotensive pregnancy.

\section{METHODS}

Comparative study of thyroid and serum lipid profile was done between those who remain normotensive and those who developed pregnancy induced hypertension in 14 to 24 weeks of gestation in the reproductive age frame of 20-35 years. Control group included pregnant females at same gestation with normal blood pressure, no proteinuria and without any other systemic or endocrine disorder. Inclusion criteria involved pregnant women (14 to 24 weeks of gestation) having blood pressure of $\geq 140 / 90 \mathrm{mmHg}$. Exclusion criteria included the cases having hypertension diagnosed before the onset of gestation, diabetes mellitus, multiple pregnancies, history of chronic hypertension, any renal disease, any metabolic disorder or medication known to affect thyroid function. Informed consents were taken from the patients and study protocol was approved by the Instituitional Ethical Committee. Samples of patients and controls were collected Beri Maternity Hospital, Amritsar and distributed in three groups viz. Group-I: non-pregnant healthy women, Group-II: normotensive pregnant women, and Group-III: hypertensive pregnant women. Serum sample was separated by centrifugation at 1500 rpm for 15 minutes, and sample was preserved for further analysis. The level of haemoglobin was analyzed by of Sahli's (acid hematin) method. ${ }^{10}$ TSH was analyzed by immunoenzymometric assay method using ERBA THYROKIT. ${ }^{11}$ The level of cholesterol was determined by CHOD-PAP method. ${ }^{12}$ Triglycerides were analyzed by GPO/POD method. ${ }^{13}$ HDL cholesterol was determined by CHOD-PAP method after precipitation with phosphotungstic acid. The level of VLDL (very low density lipoproteins) and LDL was calculated by Friedwald formula. The presence of albumin in urine was checked by using dipstick.

\section{RESULTS}

The mean age of the pregnant women included in the study was almost comparative to each other. Mean age of hypertensive pregnant women included in the study was $28.4 \pm 3.54$ years. Normotensive pregnant women and healthy women were of mean age of $26.8 \pm 2.4$ years and $28.4 \pm 3.54$ years respectively. The subjects included were of same age to achieve the comparative results. The mean weight of hypertensive pregnant women was $67.87 \pm 3.5$ $\mathrm{kgs}$, and normotensive pregnant women was $63.44 \pm 2.4$ $\mathrm{kgs}$. The healthy controls included in the study was of mean weight $61.13 \pm 3.1 \mathrm{kgs}$.

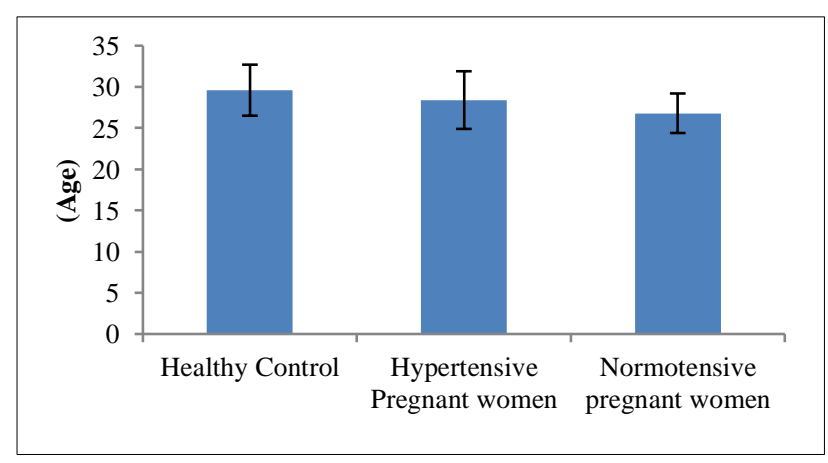

Figure 1: Mean age of hypertensive and normotensive pregnant women.

Estimation of haemoglobin level in the pregnancy is very important as the body of pregnant women requires more oxygen and the oxygen carrying capacity of the blood is proportional to the circulating haemoglobin concentration. In the present study, the haemoglobin content of hypertensive and normotensive pregnant women was found to be $11.3 \pm 4.1 \mathrm{~g} / \mathrm{dl}$ and $11.4 \pm 2.7 \mathrm{~g} / \mathrm{dl}$, 
respectively. The level of haemoglobin was found to be $10.59 \pm 1.47 \mathrm{~g} / \mathrm{dl}$ in non-pregnant healthy women. In pregnancy, the blood volume increases by $50 \%$ in the pregnancy for providing essential nutrients for developing baby. The increase in blood plasma is higher, when compared to increased RBC volume.

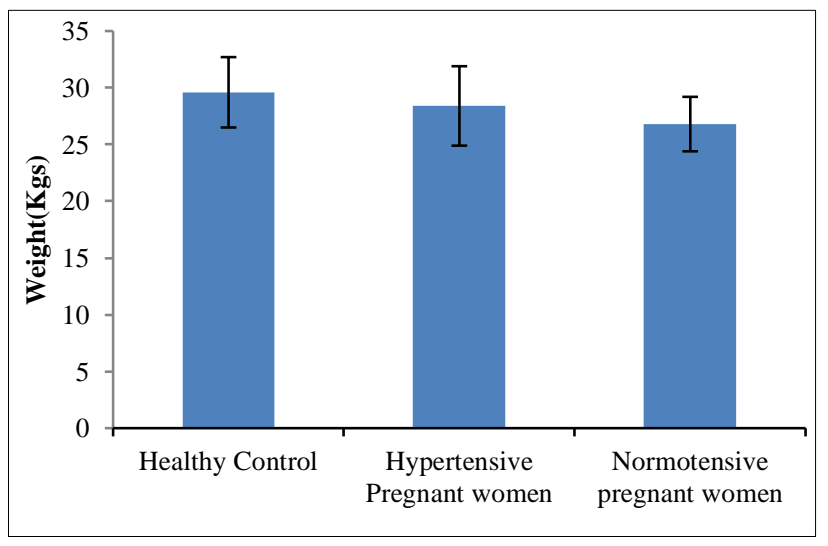

Figure 2: Mean weight of hypertensive and normotensive pregnant women.

The mean systolic and diastolic blood pressure of hypertensive pregnant women was higher as compared to normotensive pregnant women and healthy controls. In the current study, moderate hypertension was profound in the studied subjects. High blood pressure during pregnancy poses various risks including decreased blood flow to placenta, placenta abruption, intrauterine growth restriction, injury to other organs, premature delivery, and cardiovascular diseases. Severe hypertension leads to the development of preeclampsia.

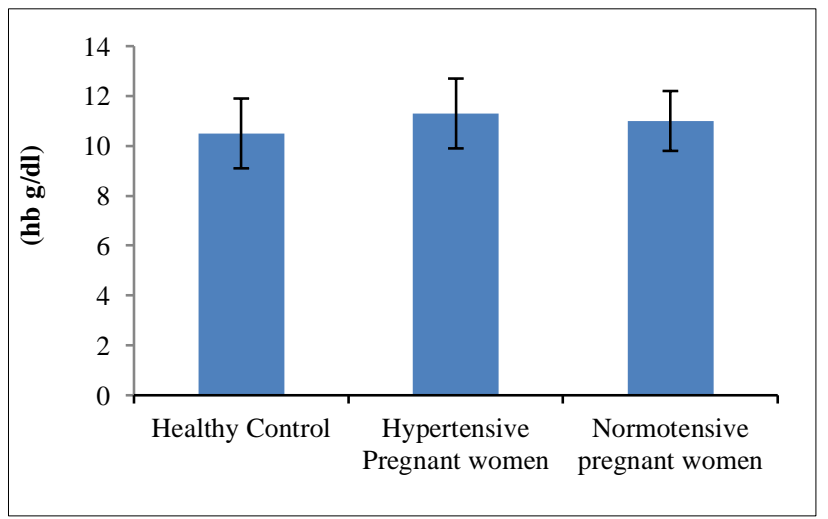

Figure 3: Haemoglobin level in hypertensive and normotensive pregnant women.

There are certain causal factors which can lead to adverse pregnancy outcomes and this is of utmost clinical importance that these should be explored and managed timely.

One such factor responsible for perinatal morbidity and mortality is maternal lipid profile. In the present study, the level of cholesterol was found higher
$(234 \pm 14.2 \mathrm{mg} / \mathrm{dl})$ in hypertensive pregnant women as compared to normotensive pregnant women $(213 \pm 19.4 \mathrm{mg} / \mathrm{dl})$ and non pregnant healthy controls $(178 \pm 16.27 \mathrm{mg} / \mathrm{dl})$. Same pattern of other lipids was found in all three cases studied. Triglycerides were found maximum $(138 \pm 16.97 \mathrm{mg} / \mathrm{dl})$ in hypertensive pregnant women, as compared to normontensive pregnant women $(118 \pm 1.55 \mathrm{mg} / \mathrm{dl})$ and non pregnant healthy controls $(114 \pm 10.9 \mathrm{mg} / \mathrm{dl})$.

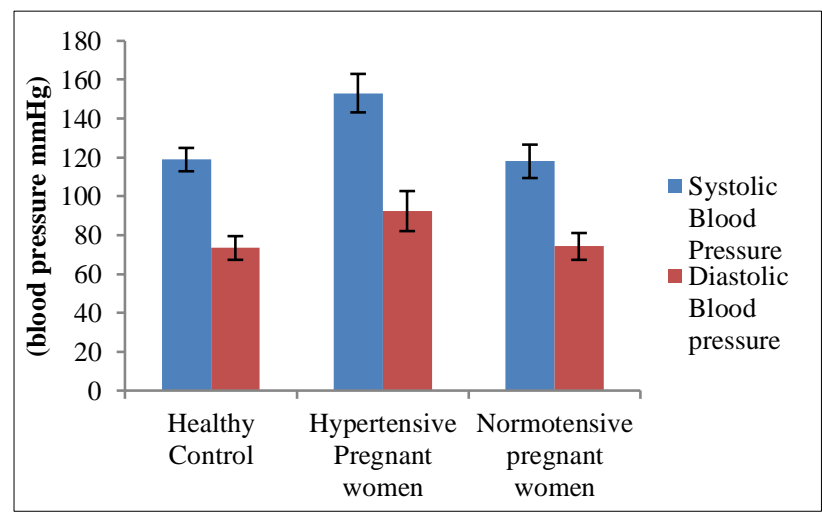

Figure 4: Systolic and diastolic blood pressure of hypertensive and normotensive pregnant women.

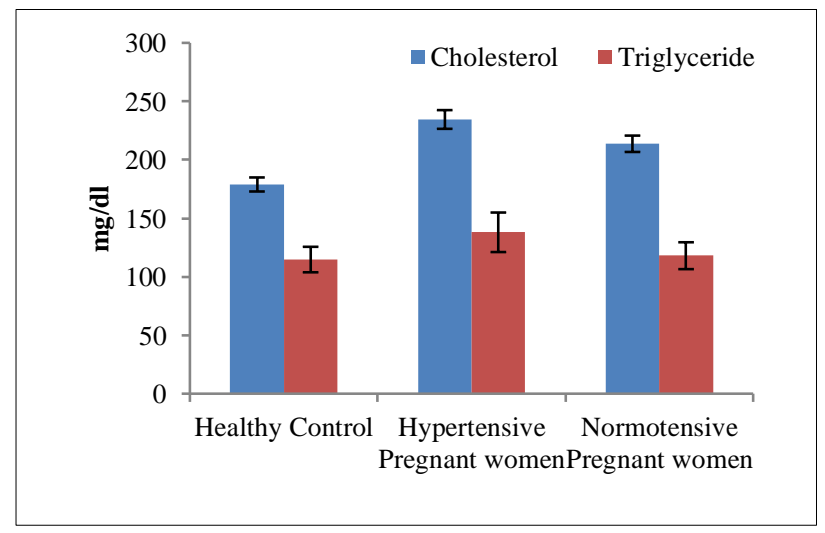

Figure 5: Lipid levels in blood serum of hypertensive and normotensive pregnant women.

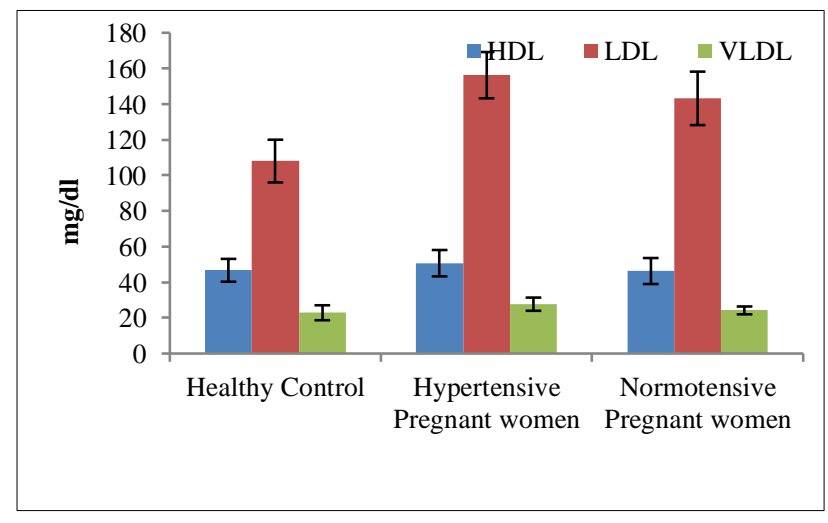

Figure 6: Levels of lipoproteins in blood serum of hypertensive and normotensive pregnant women. 
High density lipoprotein cholesterol was found almost similar in hypertensive pregnant women, normotensive pregnant women and non pregnant healthy controls. Maximum low density lipoprotein cholesterol was found in hypertensive pregnant women $(156 \pm 17.11 \mathrm{mg} / \mathrm{dl})$, followed by normotensive pregnant women, and then non-pregnant healthy controls. Very low density lipoprotein cholesterol level followed the same pattern. It can be seen from the current investigation, that high levels of lipids have been circulating in the blood of pregnant women with hypertension.

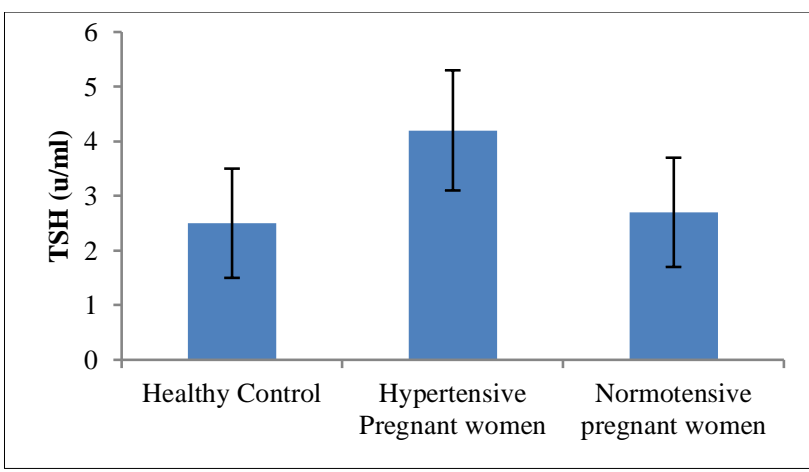

Figure 7: Level of thyroid stimulated hormone (TSH) in hypertensive and normotensive pregnant women.

\section{DISCUSSION}

Increased levels of lipids especially triglycerides were likely to be deposited in predisposed vessels and contributed to endothelial dysfunction, through the generation of low density lipoproteins, due to which vasoconstriction takes place resulting in increase in blood pressure in pregnancy. Also, estrogen induces hepatic biosynthesis of endogenous triglycerides, which are carried by VLDLc. ${ }^{14}$ Thus, VLDLc increased in the circulation due to hypertriglyceridemia as they carried endogenous triglycerides into circulation. It has also been found that enhanced LDL in pre-eclamptic patients might be due to enhanced lipid peroxidation in pregnancy. ${ }^{15}$ Dyslipidemia leads to the activation of endothelial cell, resulting in the formation of placental derived endothelial disturbing factors like lipid peroxides. These could be regarded as possible contributers for pathogenesis of pregnancy induced hypertension. ${ }^{16}$

Increased insulin sensitivity in pregnancy and increased activity of adipose tissue lipoprotein lipase (LPL) might result in endothelial dysfunction which lead to lipogenesis and dyslipidemia. ${ }^{17,18}$ Increase in lipid levels may also be due to maternal weight gain and dietary modifications. High dietary fibres can decrease triglyceride concentration and reduce preeclampsia risk. ${ }^{19}$ The rise in triglyceride rich VLDL particles during pregnancy may be due to an increased rate of synthesis caused by estrogen and decrease in rate of its removal. ${ }^{20}$ The significantly higher total cholesterol, triglyceride and LDL cholesterol in the hypertensive than in the normotensive patients in the present study is in corroboration with earlier studies. ${ }^{21}$ Researchers found significantly increased fasting serum levels of triglycerides in pre-eclamptic cases when compared with controls. $^{22}$ They found positive correlation between serum triglycerides and systolic and diastolic blood pressure in cases. For 1 unit increase in total cholesterol, triglycerides, VLDL and LDL, there were increased chances of developing hypertension by $12.6 \%, 0.3 \%$, $12.4 \%$ and $7.1 \%$ respectively. With 1 unit increase in HDL, woman has $11.4 \%$ less chance of developing hypertension in pregnancy. ${ }^{23}$ Increased cholesterol/ phospholipid ratio of the erythrocyte membrane in women with pregnancy induced hypertension has been observed in studies. ${ }^{24}$ The association of abnormal lipid values in the promotion of hypertension in pregnant women helps to investigate the underlying pathological process of hypertension in pregnancy.

The level of TSH in pregnancy is lower than normal non pregnant level since there is $50 \%$ elevation in the production of thyroid hormones $\mathrm{T}_{3}$ and $\mathrm{T}_{4}$. But in the current study, it has been observed that TSH levels in hypertensive pregnant women to be $4.26 \pm 1 \mathrm{U} / \mathrm{ml}$ which is higher than the TSH levels observed in normotensive pregnant women and non pregnant women. It can be inferred from the present study that, high blood pressure leads to the increase in TSH levels which indicated hypothyroidism. In pregnancy, thyroidal activity is increased which leads to the increase in serum TSH level. The concentration of thyroid binding globulin (TBG) might have increased during pregnancy due to the presence of high level of estrogen circulating in blood. It has been estimated that increased level of TBG in blood lead to diminish the level of triiodothyroine $\left(\mathrm{T}_{3}\right)$ and tetraiodothyroine $\left(\mathrm{T}_{4}\right)$. Decrease levels of $\mathrm{T}_{3}$ and $\mathrm{T}_{4}$ provoke pituitary gland to secrete more TSH. Moreover, increased glomerular filtration in pregnancy leads to the increase in renal clearance of iodine. The iodine loss lower the circulating level of inorganic iodine and induces a compensatory increase in thyroidal iodine clearance and its entry into the gland. Thus, it can be concluded that the cumulative effect of high blood pressure, estrogen, TBG and renal clearance leads to the hypersecretion of TSH by the gland. Maternal thyroid dysfunction during pregnancy has been associated with number of adverse outcomes. It has been suggested that there may be an existence of mutual influences between pre-eclampsia and thyroid function. ${ }^{25}$ Endothelial dysfunction is also considered an important factor in hypertensive pregnancy leading to significant increase in TSH levels in blood serum. It can be explained by excessive release of anti-angiogenic proteins from the placental to maternal blood. Low levels of free placental growth factor and free endothelial growth factor leads to endothelial dysfunction. ${ }^{26}$

In the present study, $13 \%$ of hypertensive pregnancies showed albumin excretion in urine, whereas no excretion has been observed in normotensive pregnancies and non pregnant healthy controls. It might be inferred that the 
onset of hypertension leads to the urinary albumin excretion. Preeclampsia has been defined by the combination of gestational hypertension and proteinuria of at least $0.3 \mathrm{~g} / 24 \mathrm{hrs}$, or dipstick at least 20 weeks gestation, according to the guideline to the International Society for the study of hypertension in pregnancy. Proteinuria is important sign of preeclampsia and detecting protein in urine is an integral part of the management of hypertensive pregnant women. Proteinuria in pregnancy is associated with a higher rate of maternal and foetal complications among hypertensive pregnancies. Excessive protein excretion leads to severe eclampsia which is responsible for significant morbidity and mortality in foetus, newborn infant and mother. Thus, it can be concluded that it is highly imperative to detect the condition as early as possible. Repeated urine analysis should be done to screen for this condition and should be part of standard antenatal case.

\section{CONCLUSION}

In the present study, an effort was made to explore the role of thyroid stimulating hormone and lipid profile in the pathogenesis of pregnancy induced hypertension and it was found that pregnancies had altered levels of thyroid hormone and dyslipidemia, which suggested their role in the development and pathogenesis of PIH. Therefore, early detection of thyroid abnormalities and dyslipidemia may affect the occurrence and severity of PIH and help in better management of the disease in established preeclamptic women. The levels of $\mathrm{T}_{4}$ should also be checked to identify overt or subclinical hypothyroidism. The altered levels of $\mathrm{T}_{4}$ can impair the infants neurocognitive development and also increased risks for premature birth, low birth weight and miscarriage, lower IQ levels in child. TSH levels are elevated in hypertensive pregnant women due the effect of estrogen. Among all the parameters of lipid profile, total cholesterol, TG, LDL and VLDL have been increased significantly in hypertensive pregnant women as compared to normotensive pregnant women.

\section{Funding: No funding sources}

Conflict of interest: None declared

Ethical approval: The study was approved by the Institutional Ethics Committee

\section{REFERENCES}

1. Report of the national high blood pressure education program working group on high blood pressure in pregnancy. Am J Obstet Gynecol. 2000;183:1-22.

2. Cunningham FG, Leveno KJ, Bloom SL, Dashe JS, Hoffman BL, Casey BM, Spong CY. Pregnancy hypertension. In: Kenneth J, et al., eds. Williams Obsterics. $25^{\text {th }}$ ed. New York: McGraw Hill. 2010:706.

3. Ananth CV, Keyes KM, Wapner RJ. Pre-eclampsia rates in the United States, 2010: age-period-cohort analysis. BMJ. 2013;347:6564.
4. Khan KS, Wojdyla D, Say L, Gulmezoglu AM, Van PF. WHO analysis of cause of maternal death: a systematic review. Lancet. 2006;367:1066-1074.

5. Pankaj D, Narendra M, Duru S. Principles and practice of obstetrics on gyneco. $3^{\text {rd }}$ edition. New Delhi Publication. 2008;100-103.

6. Zhu X, Cheng SY. New insights into regulation of lipid metabolism by thyroid hormone. Curr Opin Endocrinol, Diab Obes. 2010;17(5):408-13.

7. Rizos CV, Elisaf MS, Liberopoulous EN. Effects of thyroid dysfunction on lipid profile. Open Cardiovasc Med J. 2011;5:76-84.

8. Wang F, Tan Y, Wang C, Zhang X, Zhao Y, Song X. Thyroid-stimulating hormone levels within the reference range are associated with serum lipid profile independent of thyroid hormone. J Clin Endocrinal Metab. 2012;97:2724-31.

9. Dodds L, Scott H, Chandra S, Watts C. Unexplained elevated maternal serum a-feto protein and or hCG and risk of adverse outcomes. AJOG. 2003;189(3);775-81.

10. Wintrobe MM. The erythrocyte. In: Wintrobe MM, eds Clinical Hematology. 7th ed. Philadelphia: Lea and Febiger; 1975:114-115.

11. Nelson JC, Wilcox RB. Analytical performance of free and total thyroxine assays. Clinical Chemistry. 1996;42:146-154.

12. Watson D. Determination of Cholesterol. Clin Chem Acta. 1960;5(2):371-2.

13. Fossati P, Prencipe L. Serum triglycerides determined colorimetrically with an enzyme that produces hydrogen peroxide. Clin Chem. 1982;28:2077-80.

14. Glueck CJ, Fallet RW, Scheel D. Effects of oestrogenic compounds on triglyceride kinetics. Metabolism. 1975;24:537-45.

15. Wakatsuki A, Ikenoue N, Okatani Y, Shinohara K, Fukaya T. Lipoprotein particles in preeclampsia: susceptibility to oxidative modification. Obstet Gynecol. 2000;96(1):55-9.

16. Lorentsen B, Henriksen T. Plasma lipids and vascular dysfunction in pre-eclampsia. Semin Reprod Endocrinol. 1988;16(1):33-9.

17. Zhang C, Austin MA, Edwards KL. Functional variants of the lipoprotein lipase gene and the risk of preeclampsisa among non - hispanic caucasian women. Clin Genet. 2006;69:33-9.

18. Sattar N, Bendomir A, Berry C, Shepherd J, Greer IA, Packard CJ. Lipoprotein subfraction concentration in preeclampsia: pathogenic parallels to atherosclerosis. Obstet Gynecol. 1997;89(3):4038 .

19. Qiu C, Coughlin KB, Frederick IO. Dietary fiber intake in early pregnancy and risk of subsequent preeclampsia, Am J Hypertens. 2008;21:903-9.

20. Herrera E. Metabolic adaptation in pregnancy and their implications for the availability of substrates to the fetus. Eur J Clin Nutr. 2001;54:547-9. 
21. Youmbissi TJ, Djoumessi S, Nouedoui C. Profile lipidique d hypertendus camerounais noir Africians. Medicines d. Afrique Noire. 2001;31:114-8.

22. Kashinakunti S, Sunitha H, Gurupadappa K, Manjula R. Lipid profile in preeclampsia - a case control study. J Clin Diagn Res. 2010;(4):2748-51.

23. Vidyabati RK, Davina H, Singh NK. Serum $\beta$ hCG levels and lipid profile in early second trimester as predictors of pregnancy induced hypertension. $\mathbf{J}$ Obstet Gynecol. 2010;60(1):44-50.

24. Anceschi MM, Coate G, Cosmi EV, Gaiti A, Trovarelli GF. Erthrocyte membrance composition in pregnancy induced hypertension; evidence for an altered lipid profile. Int $\mathbf{J}$ Obst Gynaecol. 1992;99(6):503-7.

25. Casay BM, Dasha JS, Wells CE, Mclntire DD, Byrd W. Subclinical hypothyroidism and pregnancy outcomes. Obstet Gynecol. 2005;105:239-5.

26. Maynard SE, Karumanchi SA. Angiogenic factors and pre-eclampsia. Semin Nephrol. 2011;31(1):3346.

Cite this article as: Gill GK, Kaur M, Singh P, Kataria J. Effect of high blood pressure on thyroid and lipid profile of pregnant women. Int J Reprod Contracept Obstet Gynecol 2019;8:3699-704. 